\title{
IDEALS IN REGULAR Po Г - TERNARY SEMIGROUPS
}

\author{
U. Nagi Reddy ${ }^{1}$, G.Shobhalatha ${ }^{2}$ \\ ${ }^{1}$ Research Scholar, Department of Mathematics, Sri Krishnadevaraya University, Ananthapuramu, A.P., India-515003 \\ ${ }^{2}$ Professor, Department of Mathematics, Sri Krishnadevaraya University, Ananthapuramu, A.P., India-515003
}

\begin{abstract}
Ideals play an important role in the Algebraic structures like rings, semigroups and semirings. In this paper some generalizations of ideals in regular partially ordered (po) gamma ternary semigroups are studied. It is proved that "every ideal of a regular po gamma-ternary semigroup $T$ is semiprime" and some equivalent conditions.
\end{abstract}

Keywords: Gamma ternary semigroup, Regular, Ideal, Semiprime.

\section{INTRODUCTION}

The theory of ternary algebraic system was introduced by D.H. Lehmer [2] in 1932, but earlier such structures were studied by Kasner in1904, who gave the idea of n-ary algebras. In 1965, F.M. Sison [5] studied ideal theory in ternary semigroups. He also introduced the notions of regular ternary semigroups and characterized them by using the notion of quasi ideals. In 1981, the concept of $\Gamma$ semigroups was introduced by M.K.Sen. We know that $\Gamma$ - semigroups are generalization of semigroups. The concept partially ordered $\Gamma$-semigroup was introduced by Y.I.Know and S.K.Lee in 1961, later it has been studied by many authors. Several researchers conducted researches on the generalizations of the notion of ideals on partially ordered $\Gamma$-semigroups, which play an important role in studying of ideals on partially ordered $\Gamma$-semigroups as well as in the study of plain semigroups. In this paper, we studied different structures of ideals in po $\Gamma$ - ternary semigroups and extended these structures in regular po $\Gamma$ ternary semigroups.

Definition 1.1: A ternary semigroup $T$ is said to be a partially ordered (Po) ternary semigroup if $T$ is a partially ordered set with the relation " $\leq$ " such that $a \leq b \Rightarrow a a_{1} a_{2} \leq b a_{1} a_{2} \quad, \quad a_{1} a a_{2} \leq a_{1} b a_{2} \quad$ and $a_{1} a_{2} a \leq a_{1} a_{2} b$ for all $a, b, a_{1}, a_{2} \in T$.

Definition 1.2: A $\Gamma$ - ternary semigroup $T$ is said to be a partially ordered (Po) $\Gamma$ - ternary semigroup if $T$ is a partially ordered set with the relation " $\leq$ " such that $a \leq b \Rightarrow a \alpha a_{1} \beta a_{2} \leq b \alpha a_{1} \beta a_{2}$

$a_{1} \alpha a \beta a_{2} \leq a_{1} \alpha b \beta a_{2}$

and $a_{1} \alpha a_{2} \beta a \leq a_{1} \alpha a_{2} \beta b$ for all

$a, b, a_{1}, a_{2} \in T$ and $\alpha, \beta \in \Gamma$.

Definition 1.3: A po $\Gamma$ - ternary semigroup $T$ is said to be right (left, lateral) regular if for every $a \in T$, there exist $x, y \in T$ such that $a \leq x \alpha y \beta(a \gamma a \delta a)$ (respectively $a \leq(a \alpha a \beta a) \gamma x \delta y, \quad a \leq x \alpha(a \beta a \gamma a) \delta y \quad)$ for all $\alpha, \beta, \gamma, \delta \in \Gamma$.

Definition 1.4: A po $\Gamma$ - ternary semigroup $T$ is said to be regular if for every $a \in T$, there exist $x, y \in T$ such that $a \leq a \alpha x \beta a \gamma y \delta a$ for all $\alpha, \beta, \gamma, \delta \in \Gamma$.

Definition 1.5: Let $T$ be a po $\Gamma$ - ternary semigroup and $A$ be a non-empty subset of $T$. $A$ is called a semiprime if $a \in T, a \alpha a \beta a \in A(\alpha, \beta \in \Gamma) \Rightarrow a \in A$.

Definition 1.6: A non-empty sub set $A$ of $T$ is said to be a right (respt, left, lateral) ideal of $T$ if

1. $A \Gamma T \Gamma T \subseteq A$ respt., $T \Gamma T \Gamma A \subseteq A, T \Gamma A \Gamma T \subseteq A)$.

2. $(A] \subseteq A \quad(a \in A, b \leq a(b \in T) \Rightarrow b \in A)$.

Definition 1.7: Let $T$ be a po $\Gamma$ - ternary semigroup. Now we define a left ideal of $T$ generated by $x$ is

$$
\begin{aligned}
& L(x)=\{t \in T / t \leq x \text { or } t \leq a \alpha b \beta x \quad \text { for some } \\
& a, b \in T \text { and } \alpha, \beta, \in \Gamma\} \\
& =(x \cup T \Gamma T \Gamma x] \text { for all } x \in T .
\end{aligned}
$$

Similarly,

$$
\begin{gathered}
M(x)=\{t \in T / t \leq x \text { or } t \leq a \alpha x \beta a \text { for some } \\
a, b \in T \text { and } \alpha, \beta, \in \Gamma\}=(x \cup T \Gamma x \Gamma T] \text { is }
\end{gathered}
$$

lateral ideal generated by $x$ in $T$.

and

$$
\begin{aligned}
& R(x)=\{t \in T / t \leq x \text { or } t \leq x \alpha a \beta a \quad \text { for some } \\
& a, b \in T \text { and } \alpha, \beta, \in \Gamma\}=(x \cup x \Gamma T \Gamma T] \text { is right ideal } \\
& \text { generated by } x \text { in } T .
\end{aligned}
$$


Theorem 1.1 Every lateral ideal of a regular po $\Gamma$ - ternary semigroup $T$ is regular.

Proof: Let $\mathrm{M}$ be a lateral ideal of a regular po $\Gamma$ - ternary semigroup $T$. Then $T \Gamma M \Gamma T \subseteq M$ and $(M] \subseteq M$.

Since $T$ is regular, then $a \leq a \alpha x \beta a \gamma y \delta a$ for all $a \in T$, for some $x, y \in T$ and for all $\alpha, \beta, \gamma, \delta \in \Gamma$.

Since $T \Gamma M \Gamma T \subseteq M$ then $x \alpha a \beta y \in M$ for all $a \in M$ and $x, y \in T, \alpha, \beta \in \Gamma$.

Now we consider, $a \leq a \alpha x \beta a \gamma y \delta a$ implies that $a \leq a \alpha x \beta a \gamma y \delta a \quad=a \alpha(x \beta a \gamma y) \delta a \alpha(x \beta a \gamma y) \delta a$ $\leq a \alpha x \beta a \gamma y \delta(\operatorname{a\alpha x\beta a\gamma y\delta a)}$

$=a \alpha p \delta a \alpha p \delta a$ where $p=x \beta a \gamma y \in M$,

Hence $a \leq a \alpha p \delta a \alpha p \delta a$. Therefore $\mathrm{M}$ is a regular.

Theorem 1.2: Let $T$ be a po $\Gamma$ - ternary semigroup. Then the fowlloing are equivalent

a) $T$ is regular.

b) For any right ideal $R$, lateral ideal $M$ and Left ideal $L$ of $T,(R \Gamma M \Gamma L]=R \cap M \cap L$.

c) For any $a, b, c \in T$,

$(R(a) \Gamma M(b) \Gamma L(c)]=R(a) \cap M(b) \cap L(c)$

d) For any $a \in T$,

$(R(a) \Gamma M(a) \Gamma L(a)]=R(a) \cap M(a) \cap L(a)$.

Proof: Let $T$ be a po $\Gamma$ - ternary semigroup.

To prove that $(a) \Rightarrow(b) \Rightarrow(c) \Rightarrow(d)$

$(a) \Rightarrow(b)$ :-We assume that $T$ is regular then for any $a \in T$, and there exist $\quad x, y \in T$ such that $a \leq a \alpha x \beta a \gamma y \delta a$ for all $\alpha, \beta, \gamma, \delta \in \Gamma$.

Let $R, M$, and $L$ are right ideal, lateral ideal and left ideal in $T$ respectively.

i.e. $R \Gamma T \Gamma T \subseteq R \quad T \Gamma M \Gamma T \subseteq M$ and $T \Gamma T \Gamma L \subseteq L$ respectively.

To show that $(R \Gamma M \Gamma L]=R \cap M \cap L$.

i.e., $(R \Gamma M \Gamma L] \subseteq R \cap M \cap L$ and

$R \cap M \cap L \subseteq(R \Gamma M \Gamma L]$.

Since $R \Gamma M \Gamma L \subseteq R \Gamma T \Gamma T$,

$R \Gamma M \Gamma L \subseteq T \Gamma M \Gamma T$ and $R \Gamma M \Gamma L \subseteq T \Gamma T \Gamma L$ then $R \Gamma M \Gamma L \subseteq R \Gamma T \Gamma T \cap T \Gamma M \Gamma T \cap T \Gamma T \Gamma L$
Let $a=x \alpha y \beta z \in R \Gamma M \Gamma L$ for $x \in R, y \in M$ and $z \in L \quad$ and $\quad \alpha, \beta \in \Gamma \quad$. Then $x \alpha y \beta z \in R \Gamma T \Gamma T \cap T \Gamma M \Gamma T \cap T \Gamma T \Gamma L \quad \Rightarrow$ $a=x \alpha y \beta z \in R \cap M \cap L$

$(R \Gamma M \Gamma L] \subseteq R \cap M \cap L$

and let $a \in R \cap M \cap L$, Since $a \leq a \alpha x \beta a \gamma y \delta a$ implies that $a \leq a \alpha x \beta a \gamma y \delta a$ $\leq a \alpha x \beta a \gamma y \delta(a \alpha x \beta a \gamma y \delta a)$

$\leq(a \alpha x \beta a) \gamma(y \delta a \alpha x) \beta(a \gamma y \delta a)$

$\in(R \Gamma T \Gamma T) \Gamma(T \Gamma M \Gamma T) \Gamma(T \Gamma T \Gamma L)$

$\subseteq R \Gamma M \Gamma L$

implies that $a \in R \Gamma M \Gamma L$.

$R \cap M \cap L \subseteq(R \Gamma M \Gamma L]$

From (1) \& (2), we get

$(R \Gamma M \Gamma L]=R \cap M \cap L$.

$(b) \Rightarrow(c):-\quad$ We $\quad$ assume that $(R \Gamma M \Gamma L]=R \cap M \cap L \quad$. To prove that $(R(a) \Gamma M(b) \Gamma L(c)]=R(a) \cap M(b) \cap L(c)$ for any right ideal generated by " $a$ ", lateral ideal generated by " $b$ " and left ideal generated by " $c$ ".

For this proving, to show that $R(a) \cap M(b) \cap L(c) \subseteq(R(a) \Gamma M(b) \Gamma L(c)] \quad$ and $(R(a) \Gamma M(b) \Gamma L(c)] \subseteq R(a) \cap M(b) \cap L(c)$.

Let $x \in R(a) \cap M(b) \cap L(c)$. Then $x \in R(a), M(b)$ and $L(c)$ and we have $x \leq a$ or $x \leq a \alpha s \beta t, x \leq b$ or $x \leq s \alpha b \beta t$ and $x \leq c$ or $x \leq s \alpha t \beta c$.

Consider $x \leq a \alpha s \beta t$

$\Rightarrow x \alpha x \beta x \leq a \alpha s \beta t \alpha x \beta x$

$\leq(a \alpha s \beta t) \alpha(s \alpha b \beta t) \beta(s \alpha t \beta c)$

$\in(a \Gamma T \Gamma T) \Gamma(T \Gamma b \Gamma T) \Gamma(T \Gamma T \Gamma c)$

$\subseteq(a T T \cup\{a\})(T b T \cup\{b\})(T \Gamma T \Gamma c \cup\{c\})$

$=R(a) M(b) L(c)$

$\Rightarrow \quad x \leq x \alpha x \beta x \in R(a) M(b) L(c)$

Hence

$x \in R(a) M(b) L(c)$.

Therefore

$R(a) \cap M(b) \cap L(c) \subseteq(R(a) \Gamma M(b) \Gamma L(c)]$

$R(a) \Gamma M(b) \Gamma L(c) \subseteq R(a) \Gamma T \Gamma T \subseteq R(a)$

$R(a) \Gamma M(b) \Gamma L(c) \subseteq R(a)$ 
Similarly $\quad R(a) \Gamma M(b) \Gamma L(c) \subseteq M(a) \quad$ and

$R(a) \Gamma M(b) \Gamma L(c) \subseteq L(a)$

From (3)\&(4),

$(R(a) \Gamma M(b) \Gamma L(c)] \subseteq R(a) \cap M(b) \cap L(c)$

Therefore

$(R(a) \Gamma M(b) \Gamma L(c)]=R(a) \cap M(b) \cap L(c)$

$(c) \Rightarrow(d):-$ Assume

$(R(a) \Gamma M(b) \Gamma L(c)]=R(a) \cap M(b) \cap L(c)$

Suppose $R, M$ and $L$ are generated by " $a$ " for any $a \in T$. Then from (5) we have

$(R(a) \Gamma M(a) \Gamma L(a)]=R(a) \cap M(a) \cap L(a)$.

$(c) \Rightarrow(d)$ : - we assume

$(R(a) \Gamma M(a) \Gamma L(a)]=R(a) \cap M(a) \cap L(a)$

for any $a \in T$.

Consider

$R(a) \Gamma M(a) \Gamma L(a)=(a \Gamma T \Gamma T \cup\{a\}] \Gamma$

$(T \Gamma a \Gamma T \cup T \Gamma T \Gamma a \Gamma T \Gamma T \cup\{a\}] \Gamma(T \Gamma T \Gamma a \cup\{a\}]$

$=(a \Gamma T \Gamma T] \Gamma(T \Gamma a \Gamma T \cup T \Gamma T \Gamma a \Gamma T \Gamma T] \Gamma(T \Gamma T \Gamma a]$

$=(a \Gamma(T \Gamma T \Gamma T) \Gamma a \Gamma(T \Gamma T \Gamma T \Gamma a] \cup$

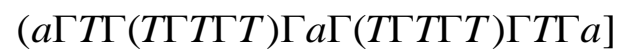

$\subseteq(a \Gamma T \Gamma a \Gamma T \Gamma a] \cup(a \Gamma T \Gamma(T \Gamma a \Gamma T) \Gamma T \Gamma a]$

$\subseteq(a \Gamma T \Gamma a \Gamma T \Gamma a] \cup(a \Gamma T \Gamma a \Gamma T \Gamma a]$

$=(a \Gamma T \Gamma a \Gamma T \Gamma a]$

Therefore $R(a) \Gamma M(a) \Gamma L(a)$

$\subseteq(a \Gamma T \Gamma a \Gamma T \Gamma a]$

Let $a \in R(a) \cap M(a) \cap L(a)$

$=(R(a) \Gamma M(a) \Gamma L(a)]$.

Then $a \in R(a) \Gamma M(a) \Gamma L(a)$

$\subseteq(a \Gamma T \Gamma a \Gamma T \Gamma a] \Rightarrow a \leq a \alpha x \beta a \gamma y \delta a]$ for Some $x, y \in T$ and for all $\alpha, \beta, \gamma, \delta \in \Gamma$. Therefore $T$ is regular.

Theorem 1.3: A po - $\Gamma$ - ternary semigroup $T$ is regular if and only if $T$ is left, right, lateral regular.
Proof: Let $T$ be regular po - $\Gamma$ - ternary semigroup. Then for any $a \in T$ and there exist $x, y \in T$ such that $a \leq a \alpha x \beta a \gamma y \delta a$ for all $\alpha, \beta, \gamma, \delta \in \Gamma$.

1. $\quad$ Consider $a \leq a \alpha x \beta a \gamma y \delta a$

$\Rightarrow a \alpha a \beta a \leq a \alpha a \beta a \alpha x \beta a \gamma y \delta a$

$=a \alpha(a \beta a \alpha x) \beta(a y y \delta a)$

$=a \alpha p \beta q$

,where $p=a \beta a \alpha x, q=a \gamma y \delta a \in T$

$\Rightarrow a \alpha a \beta a \leq a \alpha p \beta q$

$\Rightarrow a \alpha a \beta a \alpha a \beta a \leq a \alpha a \beta a \alpha p \beta q$

$\Rightarrow a \leq(a \alpha a \beta a) \alpha p \beta q$.

Therefore $T$ is left regular.

2. Consider $a \leq a \alpha x \beta a \gamma y \delta a$

$\Rightarrow a \alpha a \beta a \leq a \alpha x \beta a \gamma y \delta a \alpha a \beta a$

$=(a \alpha x \beta a) \gamma(y \delta a \alpha a) \beta a=u \alpha v \beta a$

where $u=a \alpha x \beta a, v=y \delta a \alpha a \in T$

$\Rightarrow a \alpha a \beta a \leq u \alpha v \beta a$

$\Rightarrow$ a $\alpha a \beta a \alpha a \beta a \leq u \alpha v \beta a \alpha a \beta a$

$\Rightarrow a \leq u \alpha v \beta a \alpha a \beta a$.

Therefore $T$ is right regular.

Similarly we can prove $T$ is lateral regular.

Conversely, we assume that a po - $\Gamma$ - ternary semigroup $T$ is left, right, lateral regular. Then

$a \leq(a \alpha a \beta a) \gamma x \delta y \quad, \quad a \leq x \alpha y \beta(a \gamma a \delta a) \quad$ and $a \leq x \alpha(a \beta a \gamma a) \delta y$ respectively, for all $a \in T$ for some $x, y \in T$ and for all

$\alpha, \beta, \gamma, \delta \in \Gamma$

Consider $a \leq(a \alpha a \beta a) \gamma x \delta y$

$\Rightarrow a \alpha a \beta a \leq a \alpha a \beta(a \gamma x \delta y) \alpha a \beta a=a \alpha a \beta p \alpha a \beta a$ where $p=a \gamma x \delta y \in T$.

$\Rightarrow a \alpha a \beta a \leq a \alpha a \beta p \alpha a \beta a$

$\Rightarrow$ aа $\alpha a \beta a \delta a \leq a \gamma a \alpha a \beta p \alpha a \beta a \delta a$

$\Rightarrow a \leq a a \alpha a \beta a \delta a \leq a \gamma(a \alpha a \beta p \alpha a \beta a) \delta a=a \gamma q \delta a$

$\Rightarrow a \leq a \gamma q \delta a \Rightarrow a \leq a \gamma q \delta a \leq a \gamma q \delta a a \gamma q \delta a$

$a \leq a \gamma q \delta a a \gamma q \delta a$

where $q=a \alpha a \beta p \alpha a \beta a \in T$.

Hence $T$ is regular.

Theorem 1.4: Every ideal of a regular po - $\Gamma$ - ternary semigroup $T$ is semiprime. 
Proof: Let $T$ be a regular po - $\Gamma$ - ternary

semigroup and $A$ be an ideal of $T$.

Consider $a \alpha a \beta a \in A$, for any $a \in T$,

$\alpha, \beta \in \Gamma \Rightarrow(a \alpha a \beta a) \gamma a \delta a \in A$ since $T$ is

regular.

$\Rightarrow a \leq a \alpha a \beta a \gamma a \delta a \in A \Rightarrow a \in A$.

Therefore $A$ is semiprime.

Theorem 1.5:A po $-\Gamma$ - ternary semigroup $T$ is regular if and only if it is satisfying the inequality

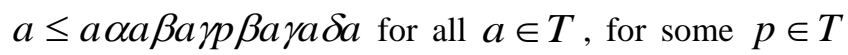
and $\alpha, \beta, \gamma, \delta \in \Gamma$

Proof: Suppose $T$ is a regular po $-\Gamma$ - ternary semigroup. Then $T$ is left, lateraland right regular. That is for any $a \in T, a \leq a \alpha a \beta a \gamma s \delta t$ and $a \leq u \alpha v \beta a \gamma a \delta a$ for some $s, t, u, v \in T$ and $\alpha, \beta, \gamma, \delta \in \Gamma$.

Since $T$ is regular then consider for any

$a \in T, a \leq a \alpha x \beta a \gamma y \delta a$ implies

$a \leq a \alpha x \beta a \gamma y \delta a$

$\leq(a \alpha a \beta a \gamma s \delta t) \alpha x \beta a \gamma y \delta a$

$\leq(a \alpha a \beta a \gamma s \delta t) \alpha x \beta a \gamma y \delta(u \alpha v \beta a \gamma a \delta a)$

$=a \alpha a \beta a \gamma(s \delta t \alpha x \beta a \gamma y \delta u \alpha v) \beta a \gamma a \delta a=a \alpha a \beta a \gamma p \beta a \gamma a \delta a$,

where $p=s \delta t \alpha x \beta a \gamma y \delta u \alpha v \in T$.

Hence $a \leq a \alpha a \beta a \gamma p \beta a \gamma a \delta a$ for every $a \in T$, for some $p \in T$ and $\alpha, \beta, \gamma, \delta \in \Gamma$.

Conversily, Consider

$a \leq a \alpha a \beta a \gamma p \beta a \gamma a \delta a$,

for every $a \in T$, for some $p \in T$ and

$\alpha, \beta, \gamma, \delta \in \Gamma$.

Now $a \leq a \alpha a \beta a \gamma p \beta a \gamma a \delta a$

$=a \alpha(a \beta a \gamma \beta$ a $\alpha a) \delta a=a \alpha z \delta a$

,where $z=a \beta a \gamma p \beta a \gamma a \in T$. Then

$a \leq a \alpha z \delta a \Rightarrow a \leq a \alpha z \delta a$

$\leq a \alpha z \delta($ a $\alpha a \beta a \gamma p \beta a \gamma a \delta a)$

$=a \alpha z \delta a \alpha(a \beta a \gamma p \beta a \gamma a) \delta a=a \alpha z \delta a \alpha z \delta a$.

i.e., $a \leq a \alpha z \delta a \alpha z \delta a$ for all $a \in T$, for

some $z \in T$ and $\alpha, \gamma, \in \Gamma$. Therefore $T$

is regular.

\section{REFERENCES}

[1] Kwon.Y.I. and Lee S.K.po $\Gamma$-semigroupsThe weakly semiprime ideal of po $\Gamma$-semigroups. KangweonKyungki Math. Jour., 5, No.2 (1997), 135-139.

[2] Lehmer, D.H : "A ternary analogue of abelian groups”, Amer. J. Math. 54 (1932) 329-338.

[3] Qing-Shun Zhu: On regular due po $\Gamma$ semigroups.International journal of pure and Applied Mathematics Valume 78 No. 4 2012, 469-478.

[4] Sen.M.K: On $\Gamma$-semigroups. Proce. Of International conference on Algebra and it's Applications. Decker Publication, New Yark (1981)301.

[5] Sison,F.M.: “ Ideal theory in ternary semigroups", Math. Japon, 10 (1965), 63-84.

[6] SukenduKar, PaltuSarkar: Fuzzy quasi ideals and fuzzy bi-ideals of ternary semigroups. Annals of Fuzzy Mathematics and Informatics Volume 4, No. 2, (October 2012) pp. 407-423 ISSN 2093-9310. 